\title{
Collective excitations of a trapped Bose-Einstein condensate in the presence of weak disorder and a two-dimensional optical lattice
}

\author{
Ying $\mathrm{Hu},{ }^{1}$ Zhaoxin Liang, ${ }^{2, *}$ and Bambi $\mathrm{Hu}^{1,3}$ \\ ${ }^{1}$ Department of Physics, Centre for Nonlinear Studies, \\ and The Beijing-Hong Kong-Singapore Joint Centre for Nonlinear and Complex Systems (Hong Kong), \\ Hong Kong Baptist University, Kowloon Tong, Hong Kong, China \\ ${ }^{2}$ Shenyang National Laboratory for Materials Science, Institute of Metal Research, \\ Chinese Academy of Sciences, Wenhua Road 72, Shenyang 110016, China \\ ${ }^{3}$ Department of Physics, University of Houston, Houston, TX 77204-5005, USA
}

(Dated: October 31, 2018)

\begin{abstract}
We investigate the combined effects of weak disorder and a two-dimensional (2D) optical lattice on the collective excitations of a harmonically trapped Bose-Einstein condensate (BEC) at zero temperature. Accordingly, we generalize the hydrodynamic equations of superfluid for a weakly interacting Bose gas in a 2D optical lattice to include the effects of weak disorder. Our analytical results for the collective frequencies beyond the mean-field approximation reveal the peculiar role of disorder, interplaying with the $2 \mathrm{D}$ optical lattice and interatomic interaction, on elementary excitations along the 3D to 1D dimensional crossover. In particular, consequences of disorder on the phonon propagation and surface modes are analyzed in detail. The experimental scenario is also proposed.

PACS numbers: 03.75.Kk,67.85.-d,03.75.Lm
\end{abstract}

\section{INTRODUCTION}

The measurement of the frequencies of collective excitations has emerged as a fundamental and precise tool to investigate the quantum many-body physics of atomic Bose-Einstein condensates (BECs) in unprecedent detail [1-4]. It not only provides an excellent confirmation of the mean-field predictions, but also represents a very effective method for probing the beyond-mean-field effects [5 7], particularly in a weakly interacting Bose gas whose condensate fraction is close to $100 \%$. Theoretically, such a gaseous BEC's system can be well represented by a single macroscopic wave function, consequently allowing for clear hydrodynamic formulations that provide analytic or semi-analytic results in describing dynamic behavior of a BEC's system.

Along this line, Stringari [5] pioneered in applying the hydrodynamic equations for superfluid to describe the ground and excited states of a magnetically trapped dilute interacting Bose gas at low temperature, yielding predictions in excellent agreement with the experiment 1]. The same scheme was later generalized by Krämer et al. [8] to include the significant effects of optical lattice, following the spectacular experimental realization of optical lattices [2, 3]. Recently, the dramatic effects caused by disorder on the quantum properties of a BEC have attracted intensive attention [9]. For example, it has been observed that even a tiny amount of disorder in the confining fields leads to a fractioning of quasi-1D condensates in waveguide structures on atom chips [10]. One particular striking effect of disorder arises from Ref.

* zhxliang@gmail.com
11 that disorder is more active in reducing superfluidity than in depleting the condensate even at zero temperature $(T=0)$. Investigations on collective modes of a BEC in a random potential using hydrodynamic scheme have been carried out by Falco et al. in Ref. [12] in lattice-free case. In view of the possibility to control both disorder and a BEC in an optical lattice almost at will [13], an important direction consists in investigating the collective excitations of a trapped BEC in the combined presence of disorder and optical lattice.

Another significant influence of disorder on Bose gases is that it enhances quantum fluctuations and the beyondmean-field corrections thereof to the equations of state. Such corrections in the free space arising from interatomic interaction have been predicted by Lee, Huang and Yang (LHY) [14]. Yet measuring these corrections remains challenging, as they are usually too small to be observed. Pitaevskii and Stringari [6] first pointed out observing these beyond-mean-field effects in the frequency shift of collective excitations. Later in Ref. [15], Orso et al. suggested that these frequency shift could be further magnified by introducing optical lattice to enhance correlations. Furthermore, Ref. [12] showed that disorder also caused a frequency shift in collective modes, with a magnitude comparable to interatomic interaction. It is therefore expected that the combined presence of disorder and optical lattice might considerably enhance the quantum fluctuations, ultimately rendering the effects beyond mean field more observable in the experiment. On the other hand, disorder has been shown to cause a departure from Kohn's theorem for the dipole mode [12], a signature to distinguish the effects beyond mean field due to disorder from that due to interatomic interaction. An especially appealing question, therefore, consists in exploring the beyond-mean-field effects arising 
from the interplay between disorder and optical lattice on the collective excitations of a trapped BEC.

Hence, we are motivated to launch a comprehensive investigation on a trapped BEC, taking into account of the combined effects of disorder, optical lattice and interatomic interaction. Against this background, a 2D optical lattice is especially favorable, because it induces a characteristic $3 \mathrm{D}$ to $1 \mathrm{D}$ dimensional crossover in the quantum fluctuations of a BEC [15], even in the presence of weak disorder [16]. Loading a BEC into a 2D optical lattice, therefore, provides an effective tool to study a 1D BEC and 3D BEC through asymptotic analysis [15, 16].

In this paper, we focus on studying the collective excitations of a harmonically trapped BEC in the presence of weak disorder and a $2 \mathrm{D}$ optical lattice at $T=0$. Accordingly, we extend the hydrodynamic equations for superfluid in a $2 \mathrm{D}$ optical lattice to include the effect of weak disorder. Using the developed hydrodynamic equations, we calculate the collective frequencies beyond the mean-field approximation. Our analytical results reveal the peculiar role of disorder, when interplaying with a $2 \mathrm{D}$ optical lattice and interatomic interaction, on the excited states of a trapped BEC along the $3 \mathrm{D}$ to $1 \mathrm{D}$ dimensional crossover. In particular, the effects of disorder on sound velocity and surface modes are discussed in detail. We also propose possible experimental conditions to realize our scenario.

The outline of this paper is as follows. In Sec. II, we introduce the Hamiltonian for a magnetically trapped BEC in the presence of a 2D optical lattice and weak disorder. We first examine the case in the absence of disorder in Sec. III where we follow the scheme in [8] for generalizing hydrodynamic equations in Ref. [5] to include the effects of a $2 \mathrm{D}$ optical lattice. Thereafter in Sec. IV, we account for the presence of random potential and extend the results in Sec. III to include the effects of weak disorder. Using these equations, we analyze in Sec. V the combined effects of disorder and a 2D optical lattice on the collective excitations of a trapped BEC. In particular, we calculate the beyond-mean-field corrections to the collective frequencies. Detailed discussions on the sound velocity and low-lying modes as well as quantum behaviors along the dimensional crossover are presented. Finally in Sec. VI, we summarize our results and propose the experimental conditions for realizing our scenario.

\section{HAMILTONIAN FOR A TRAPPED BEC IN THE PRESENCE OF WEAK DISORDER AND A 2D OPTICAL LATTICE}

The N-body Hamiltonian describing the Bose system at $T=0$ has the form [1]

$$
\begin{aligned}
H-\mu N=\int d \mathbf{r} \hat{\Psi}^{\dagger}(\mathbf{r})[ & -\frac{\hbar^{2} \nabla^{2}}{2 m}-\mu+V_{e x t}(\mathbf{r})+V_{\text {ran }}(\mathbf{r}) \\
& \left.+\frac{g}{2} \hat{\Psi}^{\dagger}(\mathbf{r}) \hat{\Psi}(\mathbf{r})\right] \hat{\Psi}(\mathbf{r})
\end{aligned}
$$

where $\hat{\Psi}(\mathbf{r})$ is the field operator for bosons with mass $m$, $\mu$ is the chemical potential, $\hat{N}=\int d \mathbf{r} \hat{\Psi}^{\dagger}(\mathbf{r}) \hat{\Psi}(\mathbf{r})$ is the number operator, and $g=4 \pi \hbar^{2} a / m$ is the coupling constant with $a$ being the $s$-wave scattering length in the free space. In Hamiltonian (1), $V_{\text {ran }}(\mathbf{r})$ and $V_{\text {ext }}(\mathbf{r})$ respectively represent the random potential and the trapping potential.

The external trapping potential $V_{\text {ext }}(\mathbf{r})$ in Hamiltonian (1) is generated by a superposition of a $3 \mathrm{D}$ harmonic confinement $V_{h o}(\mathbf{r})$ of magnetic origin and a $2 \mathrm{D}$ optical lattice $V_{\text {opt }}(\mathbf{r})$ modulated along the $x-y$ plane

$$
\begin{aligned}
V_{\text {ext }}(\mathbf{r}) & =\frac{1}{2} m\left(\omega_{x}^{2} x^{2}+\omega_{y}^{2} y^{2}+\omega_{z}^{2} z^{2}\right) \\
& +s E_{R}\left[\sin ^{2}\left(q_{B} x\right)+\sin ^{2}\left(q_{B} y\right)\right],
\end{aligned}
$$

where $\omega_{x}, \omega_{y}$ and $\omega_{z}$ are the frequencies of the harmonic trap, $s$ is a dimensionless factor labeled by the intensity of laser beam and $E_{R}=\hbar^{2} q_{B}^{2} / 2 m$ is the recoil energy with $\hbar q_{B}$ being the Bragg momentum. The lattice period is fixed by $d=\pi / q_{B}$.

Disorder $V_{\text {ran }}(\mathbf{r})$ in Hamiltonian (1) is produced by the random potential associated with quenched impurities [11, 16, 17]

$$
V_{\text {ran }}(\mathbf{r})=\sum_{i=1}^{N_{i m p}} v\left(\left|\mathbf{r}-\mathbf{r}_{\mathbf{i}}\right|\right),
$$

with $v(\mathbf{r})$ describing the two-body interaction between bosons and impurities, $\mathbf{r}_{i}$ being the randomly distributed positions of impurities and $N_{i m p}$ counting the number of $\mathbf{r}_{i}$. To obtain the concrete form of the pair potential $v(\mathbf{r})$, we need to investigate the scattering problem between a boson with mass $m$ and a quenched impurity with mass $M$ in the presence of a $2 \mathrm{D}$ optical lattice [18]. Here, we restrict ourself to the conditions of a dilute BEC system in the presence of a very small concentration of disorder. Thereby, the potential $v(\mathbf{r})$ can be expressed by an effective pseudo-potential $v(\mathbf{r})=\tilde{g}_{i m p} \delta(\mathbf{r})$ [11]. For the sake of notation convenience, one can write $\tilde{g}_{i m p}=2 \pi \hbar^{2} \tilde{b} / m$ $[11,16,17]$. Here, $\tilde{b}$ effectively characterizes the latticemodified strength of disorder, while the reduced mass for the boson-impurity pair coincides with $m$ when the mass of impurity is taken to be infinite due to its quenched nature [17], i.e. $\lim _{M \rightarrow \infty} m M /(M+m)=m$. 


\section{MACROSCOPIC DYNAMICS OF A BEC LOADED IN A 2D OPTICAL LATTICE}

Let us first consider the absence of disorder $\left(V_{\text {ran }}=0\right)$ in Hamiltonian (1). In this section we shall follow the scheme in Ref. 8] and reproduce corresponding results for generalizing the hydrodynamic equations of superfluid [5] to include the effects of a $2 \mathrm{D}$ optical lattice at $T=0$. The validity condition for the presented hydrodynamic formulation requires relatively large $s$ where the interwell barriers are significantly larger than the chemical potential, while quantum tunneling is still sufficient to ensure full coherence [5, 8].

Under above conditions, a formulation for the macroscopic dynamics can be accomplished by considering the long-wavelength limit of Hamiltonian (11) and the action functional thereof for the model system. To this end, we start from the ansatz for the condensate order parameter within the tight-binding approximation [8, 15]

$$
\Phi(\mathbf{r})=\sum_{l_{x}, l_{y}} W\left(x-l_{x} d\right) W\left(y-l_{y} d\right) f_{l_{x}, l_{y}}(z) e^{i S_{l_{x}, l_{y}}(z)} .
$$

Here, $S_{l_{x}, l_{y}}$ is the phase of the $\left(l_{x}, l_{y}\right)$ component of the order parameter, while $W\left(x-l_{x} d\right), W\left(y-l_{y} d\right)$ and $f_{l_{x}, l_{y}}(z)$ are real functions. In addition, the $W\left(x-l_{x} d\right)$ and $W\left(y-l_{y} d\right)$ are further assumed to satisfy the periodic conditions, i.e. $W\left(x-l_{x} d\right)=W_{0}(x)$ and $W(y-$ $\left.l_{y} d\right)=W_{0}(y)$ where both $W_{0}(x)$ and $W_{0}(y)$ are localized at the origin. A transcription to the continuum limit is achieved by coarse-graining through the replacement $\sum_{l_{x}, l_{y}} \rightarrow\left(1 / d^{2}\right) \int d x d y$. One thereby introduces the smoothed phase $S\left(S_{l}(z) \rightarrow S(\mathbf{r})\right)$ and a coarse-grained density [8]

$$
n(\mathbf{r})=\frac{1}{d^{2}} f_{l_{x}, l_{y}}^{2}(z),
$$

with $x=l_{x} d, y=l_{y} d$.

The long-wavelength limiting form of the Lagrangian functional can then be obtained by substituting ansatz (41) into Hamiltonian (11), applying coarse-graining and proceeding as Ref. [8]:

$$
\begin{aligned}
L & =\left\langle-i \hbar \int \Phi^{*} \frac{\partial}{\partial t} \Phi d \mathbf{r}+H-\mu N\right\rangle \\
& =\int d \mathbf{r}\left[\hbar \frac{\partial S}{\partial t}-2 t\left[\cos \left(d \partial_{x} S\right)+\cos \left(d \partial_{y} S\right)\right]\right. \\
& \left.+\frac{\hbar^{2}}{2 m}\left(\partial_{z} S\right)^{2}+V_{h o}(\mathbf{r})+\frac{\tilde{g}}{2} n(\mathbf{r})-\mu\right] n(\mathbf{r}),
\end{aligned}
$$

where

$$
t=-\int d x W_{0}(x)\left[-\frac{\hbar^{2}}{2 m} \partial_{x}^{2}+V_{\text {opt }}(x)\right] W_{0}(x-d)
$$

relates to the tunneling rate between adjacent walls with $V_{\text {opt }}(x)=s E_{R} \sin ^{2}\left(q_{B} x\right)$ being the external optical lattice applied along $x$ direction, and $\tilde{g}=4 \pi \hbar^{2} \tilde{a} / m$ is the lattice-renormalized coupling constant with $\tilde{a}=C^{2} a$ and $C=d \int_{-d / 2}^{d / 2} W_{0}^{4}(x) d x$. In addition, Eq. (6) is derived based on the Thomas-Fermi (TF) approximation [5] in the large $N$ limit where one can neglect the quantum pressure terms originating from the radial term in the kinetic energy [8].

With Eq. (6), the equations of motion are derived by requiring that the action $A=\int_{0}^{t} L d t$ be stationary under arbitrary variations in the macroscopic density $n(\mathbf{r})$ and the phase $S(\mathbf{r})$ with fixed endpoints, i.e. $\delta A / \delta n=0$ and $\delta A / \delta S=0$. The resulting equations are [19]

$$
\begin{aligned}
\frac{\partial n}{\partial t}+\frac{2 t d}{\hbar} \partial_{\perp}\left[n \sin \left(d \partial_{\perp} S\right)\right]+\frac{\hbar}{m} \partial_{z}\left[n \partial_{z} S\right] & =0, \\
\hbar \frac{\partial S}{\partial t}+\delta \mu-2 t \cos \left(d \partial_{\perp} S\right)+\frac{\hbar^{2}}{2 m}\left(\partial_{z} S\right)^{2} & =0,
\end{aligned}
$$

where $\delta \mu=V_{h o}+\tilde{g} n-\mu$ is the change of the chemical potential with respect to its ground state value 16]. In Eqs. (8) and (91), two notations have been introduced for simplicity: $\partial_{\perp}\left[n \sin \left(d \partial_{\perp} S\right)\right] \equiv$ $\partial_{x}\left[n \sin \left(d \partial_{x} S\right)\right]+\partial_{y}\left[n \sin \left(d \partial_{y} S\right)\right]$ and $\cos \left(d \partial_{\perp} S\right) \equiv$ $\cos \left(d \partial_{x} S\right)+\cos \left(d \partial_{y} S\right)$. Accordingly, one can define the superfluid velocity $\mathbf{v}$ in a 2 D optical lattice as [8]

$$
\begin{aligned}
v_{x(y)} & =\frac{\hbar}{m^{*}} \partial_{x(y)} S, \\
v_{z} & =\frac{\hbar}{m} \partial_{z} S,
\end{aligned}
$$

where the effective mass $m^{*}$ is introduced by

$$
\frac{m}{m^{*}}=\frac{2 m t d^{2}}{\hbar^{2}}
$$

which accounts for the increased inertia of the system along the direction of optical lattice [20, 21]. The ground state density within the TF approximation is obtained by putting $\delta \mu=0$ that yields $[5,[8]$ :

$$
n_{T F}(\mathbf{r})=\frac{1}{\tilde{g}}\left[\mu-\frac{1}{2} m\left(\omega_{x}^{2} x^{2}+\omega_{y}^{2} y^{2}+\omega_{z}^{2} z^{2}\right)\right] .
$$

Eq. (13) shows a fattened cloud in the presence of a lattice due to increased repulsive interaction characterized by $\tilde{g}$.

In the limit of small oscillations, Eqs. (8) and (9) can be linearized by substitutions of Eqs. (10) and (11) and decomposing $n(\mathbf{r})=n_{T F}(\mathbf{r})+\delta n(\mathbf{r})$ and $\mathbf{v}(\mathbf{r})=\delta \mathbf{v}(\mathbf{r})$. The resulting equations read,

$$
\begin{aligned}
& \frac{\partial \delta n}{\partial t}+\nabla \cdot\left[n_{T F} \delta \mathbf{v}\right]=0 \\
& \hbar \frac{\partial \delta S}{\partial t}+\delta \mu=0
\end{aligned}
$$

With Eqs. (10) and (11), one can combine Eqs. (14) and (15) and consequently obtain the equation for the propagation of a density disturbance in the model system at $T=0$,

$$
m \frac{\partial^{2} \delta n}{\partial t^{2}}-\widetilde{\nabla} \cdot\left[n_{T F} \tilde{\nabla} \delta \mu\right]=0
$$


where we have introduced the notion [15]

$$
\widetilde{\nabla} \equiv\left(\widetilde{\nabla}_{\perp}, \nabla_{z}\right)=\left(\sqrt{\frac{m}{m^{*}}} \frac{\partial}{\partial x}, \sqrt{\frac{m}{m^{*}}} \frac{\partial}{\partial y}, \frac{\partial}{\partial z}\right) .
$$

Eq. (16) bears formal resemblance with the corresponding equation in Ref. [5] for small-amplitude density fluctuation in the free space. Hence by assuming that the effect of optical lattice is captured by the effective mass $m^{*}$ and the renormalized interaction $\tilde{g}$, one can study the quantum behavior of a BEC as if the space is homogeneous in the absence of harmonic trap, despite the application of periodic potential. In particular, in the limit $8 t \gg \mu$ where the system retains a 3D behavior, Ref. [22] has experimentally verified the validity of the above mass renormalization theory. In the opposite $1 \mathrm{D}$ regime, on the other hand, the use of hydrodynamic equations is justified by the superfluidity of the quasi-1D tubes [23] and by the avoidance of the Mott-insulator phase.

\section{MACROSCOPIC DYNAMICS OF A BEC IN THE PRESENCE OF WEAK DISORDER AND A 2D OPTICAL LATTICE}

In this section, we take into account of $V_{\text {ran }}(\mathbf{r}) \neq 0$ in Hamiltonian (11) and investigate the effects of weak disorder, combined with a 2D optical lattice, on elementary excitations of a trapped BEC at $T=0$. To this purpose, we generalize corresponding hydrodynamic scheme in Sec. III to include the effects of weak external randomness.

The disorder in this paper is associated with quenched impurities which generate a random potential $V_{\text {ran }}(\mathbf{r})$ given by Eq. (3). For simplicity, we further assume that the randomness is uniformly distributed with density $n_{i m p}=N_{i m p} / V$ and Gaussian correlated [17]. Hence, the two basic statistical properties of disorder, in the presence of a $2 \mathrm{D}$ optical lattice, are the average value $\left\langle V_{0}\right\rangle=\tilde{g}_{i m p} n_{i m p}$ and the correlation function

$$
\left\langle V_{\mathbf{k}} V_{-\mathbf{k}}\right\rangle=\frac{1}{V} \tilde{g}_{i m p}^{2} n_{i m p}
$$

where $\tilde{g}_{i m p}=2 \pi \hbar^{2} \tilde{b} / m$ is the lattice-renormalized bosonimpurity interaction, $n_{i m p}$ is the impurity concentration, and $V_{\mathbf{k}}=(1 / V) \int e^{i \mathbf{k} \cdot \mathbf{r}} V_{\text {ran }}(\mathbf{r}) d \mathbf{r}$ is the Fourier transform of $V_{\text {ran }}(\mathbf{r})$. Here, the notation $\langle\ldots\rangle$ stands for the ensemble average over all possible disorder configurations [28]. The effect of disorder in a lattice can be characterized by two important parameters: the concentration of disorder $\kappa=n_{i m p} / n$ and the ratio of effective interaction strength

$$
\tilde{R}=\frac{n_{i m p}}{n} \frac{\tilde{b}^{2}}{\tilde{a}^{2}}
$$

The validity conditions for following hydrodynamic formulation in a random potential require the correlation length of disorder to be much smaller than the healing length of superfluid [12], in addition to those required in Sec. III.

\section{A. Hydrodynamic equations}

We start from Hamiltonian (1) with $V_{\text {ran }}(\mathbf{r})$ given by Eq. (3) within the white noise approximation [17], and follow similar procedures in Sec. III. The resulting hydrodynamic equation for the macroscopic phase $S$ reads

$\hbar \frac{\partial S}{\partial t}+\delta \mu-2 t\left[\cos \left(d \partial_{x} S\right)+\cos \left(d \partial_{y} S\right)\right]+\frac{\hbar^{2}}{2 m}\left(\partial_{z} S\right)^{2}=0$

where $\delta \mu=V_{h o}+\tilde{g} n-\mu$ is the change of chemical potential with respect to its ground state value. Compared to Eq. (9) in the absence of disorder $\left(V_{\text {ran }}=0\right)$, Eq. (20) takes similar form, except that the associated chemical potential $\mu$ here includes the dramatic effects of disorder on the system's ground state [11, 16, 26]. The presence of disorder, therefore, only affects the dynamics of phase fluctuation indirectly through the equation of state. We will determine $\mu$ in the following section. Once Eq. (20) is solved for $S$, the superfluid velocity $\mathbf{v}$ can be obtained from Eqs. (10) and (11). In the limit of small-amplitude phase fluctuation $S=\delta S$, Eq. (20) can be linearized as

$$
\hbar \frac{\partial \delta S}{\partial t}+\delta \mu=0
$$

Here, the hydrodynamical variables are assumed to be ensemble averages over all possible realizations of external disorder. By this assumption, the validity of Eqs. (20) and (21) should be restricted to the self-averaging regime where the wavelength of the hydrodynamic modes is much larger than the correlation length of disorder [12].

For the equation of total density $n(\mathbf{r})$, the corresponding conservation law is unaffected by the presence of weak disorder. However, the random potential gives rise to a normal component in the fluid [11, 12, 16, 26] which is pinned by quenched impurities and is neither a fluid nor dynamic. Thus, when a small perturbation $\delta \mathbf{v}$ is applied, only superfluid component responds to the probe and contributes to the induced current $\mathbf{j}=n_{s} \delta \mathbf{v}$ with $n_{s}$ being the superfluid density [26, 28], whereas the normal component remains stationary. Consistent with this picture, a density disturbance $\delta n(\mathbf{r})$ from the equilibrium density profile is related to the perturbation $\delta \mathbf{v}$ through the equation of continuity

$$
\frac{\partial \delta n}{\partial t}+\nabla \cdot\left[n_{s} \delta \mathbf{v}\right]=0 .
$$

Here, $\delta \mathbf{v}$ is determined from $\delta S$ through Eqs. (10) and (11). Hence Eq. (22) can be combined with Eq. (21) to yield the hydrodynamic equation

$$
m \frac{\partial^{2} \delta n}{\partial t^{2}}+\tilde{\nabla} \cdot\left[n_{s} \tilde{\nabla} \delta \mu\right]=0
$$

where the notation $\tilde{\nabla}$ is given by Eq. (17).

Eq. (23) is one of the main results of this paper. This equation is characterized by the disorder-depleted superfluid density $n_{s}$, the renormalized interaction $\tilde{g}$ and an 
affective mass $m^{*}$ accounting for the presence of optical lattice. For vanishing disorder, the whole system participates superflow and Eq. (23) becomes the corresponding equation for superfluid in Refs. [8, 15]; whereas for vanishing optical lattice, our result recovers the corresponding one in Ref. [12]; while for vanishing optical lattice as well as disorder, Eq. (23) passes to that in Ref. [5] for superfluid in harmonic traps. We emphasize that Eq. (23) has no similarity with the corresponding phenomenological equations in Landau's two-fluid theory [24]. The disorder induced normal component is not dynamic. It's effect on the dynamics of superfluid is indirectly exhibited through the superfluid density $n_{s}=n-n_{n}$ and the equation of state.

\section{B. Equations of state beyond the mean-field approximation}

Eq. (23) needs to be supplemented with prescriptions for the chemical potential $\mu(\mathbf{r})$, the ground state density $n(\mathbf{r})$ and the superfluid density $n_{s}(\mathbf{r})$. In what follows, we derive expressions for these quantities based on the local density approximation (LDA) [5].

\section{Chemical potential}

For the density profile that varies on a macroscopic scale, one can invoke the LDA for the chemical potential

$$
\mu(\mathbf{r})=\mu_{l}[n(\mathbf{r})]+V_{h o}(\mathbf{r}),
$$

where $\mu_{l}$ refers to the chemical potential calculated for a BEC's system with Hamiltonian (11) in the absence of harmonic trap $\left(V_{h o}=0\right)$.

The $\mu_{l}$ can be determined from $\mu_{l}=\partial E_{g} / \partial N$, with $E_{g}$ representing the ground state energy of the model system without harmonic trap. To calculate $E_{g}$ microscopically, we adopt Bogoliubov's theory [16] and expand the field operators in Hamiltonian (1) in the form $\hat{\Psi}(\mathbf{x})=\sum_{\mathbf{k}} \hat{a}_{\mathbf{k}} e^{-i k_{z} z} \phi_{k_{x}}(x) \phi_{k_{y}}(y)$, where $\phi_{k_{x}}(x) \phi_{k_{y}}(y)$ corresponds to the lowest Bloch band of the BEC system under consideration. In the tight-binding approximation, one can write $\phi_{k_{x}}(x)=\sum_{l} e^{i l k_{x}} w(x-$ ld) with $w(x)=\exp \left[-x^{2} / 2 \sigma^{2}\right] / \pi^{1 / 4} \sigma^{1 / 2}$ and $d / \sigma \simeq$ $\pi s^{1 / 4} \exp (-1 / 4 \sqrt{s})$ [8, 16]. The dominate presence of condensate is taken into account through the approximation $\hat{a}_{\mathbf{k}} \approx \sqrt{n V} \delta_{\mathbf{k} 0}+\delta \hat{a}_{\mathbf{k}}$. Retaining only the quadratic terms in the excitations $\delta \hat{a}_{\mathbf{k}}$ and $\delta \hat{a}_{\mathbf{k}}^{\dagger}$ from the condensate, the truncated Hamiltonian (1) can be ultimately diagonalized by the Bogoliubov transformation [16]: $\hat{a}_{\mathbf{k}}=u_{\mathbf{k}} \hat{c}_{\mathbf{k}}-v_{\mathbf{k}} \hat{c}_{-\mathbf{k}}^{\dagger}-\sqrt{N} V_{k}\left(u_{\mathbf{k}}-v_{\mathbf{k}}\right)^{2} / E_{\mathbf{k}}$ and $\hat{a}_{\mathbf{k}}^{\dagger}=u_{\mathbf{k}} \hat{c}_{\mathbf{k}}^{\dagger}-v_{\mathbf{k}} \hat{c}_{-\mathbf{k}}-\sqrt{N} V_{-k}\left(u_{\mathbf{k}}-v_{\mathbf{k}}\right)^{2} / E_{\mathbf{k}}$ with $v_{\mathbf{k}}^{2}=u_{\mathbf{k}}^{2}-1=\left[\left(\varepsilon_{k}^{0}+\tilde{g} n\right) / E_{\mathbf{k}}-1\right] / 2$ and $E_{\mathbf{k}}=$ $\sqrt{\varepsilon_{k}^{0}\left(\varepsilon_{k}^{0}+2 \tilde{g} n\right)}$. Here, $V_{\mathbf{k}}$ is the Fourier transform of $V_{\text {ran }}(\mathbf{r})$ and $\epsilon_{\mathbf{k}}^{0}=\hbar^{2} k_{z}^{2} / 2 m+2 t\left[2-\cos \left(k_{x} d\right)-\cos \left(k_{y} d\right)\right]$ is the energy dispersion of a noninteracting Bose gas in the presence of a 2D optical lattice. Consequently, one finds the ground state energy $E_{g}=\tilde{g} n^{2} V / 2-\sum_{k \neq 0}\left(\varepsilon_{\mathbf{k}}^{0}+\tilde{g} n-\right.$ $\left.E_{\mathbf{k}}\right) / 2+N\left[\tilde{g}_{i m p} n_{i m p}-\left(\tilde{g}_{i m p}^{2} n_{i m p} / V\right) \sum_{\mathbf{k} \neq \mathbf{0}} \varepsilon_{\mathbf{k}}^{0} / E_{\mathbf{k}}^{2}\right]$.

The resulting $\mu_{l}$ in Eq. (24), in a convenient form, is given by

$$
\mu_{l}(n)=\tilde{g} n\left[1+k_{i n t}(n)+k_{d i s}(n)\right],
$$

which includes the first order correction to the result $\mu=\tilde{g} n$ in the mean-field approximation. Precisely, interatomic interaction and weak disorder respectively give rise to the beyond-mean-field corrections

$$
k_{\text {int }}(n)=\frac{1}{4 \pi \hbar} \frac{\sqrt{2 m \tilde{g} n}}{n d^{2}}\left[x \frac{d f(x)}{d x}-\frac{3}{2} f(x)\right]
$$

and

$$
k_{d i s}(n)=\kappa \frac{\tilde{b}}{\tilde{a}}+\frac{\tilde{R}}{4 \pi \hbar} \frac{\sqrt{m \tilde{g} n}}{n d^{2}}\left[x \frac{d Q(x)}{d x}-\frac{3}{2} Q(x)\right],
$$

with $x=2 t / \tilde{g} n$ and $\kappa=n_{i m p} / n$. Here, the $f(x)$ and $Q(x)$ as functions of variable $x$ are respectively defined as [16]

$$
f(x)=\frac{\pi}{2 \sqrt{x}} \int_{-\pi}^{\pi} \frac{d^{2} \mathbf{k}}{(2 \pi)^{2}} \frac{{ }_{2} F_{1}\left(\frac{1}{2}, \frac{3}{2}, 3,-\frac{2}{x \gamma(\mathbf{k})}\right)}{\sqrt{\gamma(\mathbf{k})}},
$$

and

$$
Q(x)=\frac{\pi}{2} \int_{-\pi}^{\pi} \frac{d k_{x}}{2 \pi} \frac{{ }_{2} F_{1}\left(\frac{1}{2}, \frac{1}{2}, 1, \frac{x^{2}}{\left(1+x+x \sin ^{2}\left(k_{x} / 2\right)\right)^{2}}\right)}{\sqrt{x \sin ^{2}\left(k_{x} / 2\right)+1}} .
$$

In Eq. (28), $\gamma(\mathbf{k})=2-\cos \left(k_{x}\right)-\cos \left(k_{y}\right)$. The function ${ }_{2} F_{1}(a, b, c, d)$ in Eqs. (28) and (29) is the hypergeometric function [31] and the integration over the transverse quasimomenta is restricted to the first Brillouin zone, i.e. $\left|k_{x}\right|,\left|k_{y}\right| \leq \pi$ in Eq. (28) and $\left|k_{x}\right| \leq \pi$ in Eq. (29).

The parameter $x=2 t / \tilde{g} n$ controls the dimensional crossover for a uniform gas $\left(V_{t r}=0\right)$. In the limit $x \rightarrow 0$, corresponding to $8 t \ll \mu_{l}$, the system undergoes a dimensional crossover to a $1 \mathrm{D}$ regime where the $f(x)$ saturates to the value $4 \sqrt{2} / 3$. In this limit, we can neglect the Bloch dispersion and Eq. (25) approaches asymptotically to the chemical potential of a $1 \mathrm{D}$ Bose gas in the presence of weak disorder

$$
\begin{aligned}
\mu_{l}(n) & =\tilde{g} n\left\{1-\frac{1}{\pi \hbar} \frac{\sqrt{m \tilde{g} n}}{n d^{2}}\right. \\
& \left.+\kappa \frac{\tilde{b}}{\tilde{a}}+\frac{\tilde{R}}{4 \pi \hbar} \frac{\sqrt{m \tilde{g} n}}{n d^{2}}\left[x \frac{d Q(x)}{d x}-\frac{3}{2} Q(x)\right]\right\}
\end{aligned}
$$

Eq. (30) effectively generalizes the Lieb-Linger solution of the 1D model expanded in the weak coupling regime to include the effects of weak disorder [16].

In the opposite limit $x \gg 1$, corresponding to $8 t \gg \mu_{l}$, the system retains an anisotropic $3 \mathrm{D}$ behavior. In this 
regime, the functions (28) and (29) respectively reach the asymptotic law $f(x) \simeq 1.43 / \sqrt{x}-16 \sqrt{2} / 15 \pi x$ and $Q(x) \simeq-1 / \sqrt{\pi} x$. Eq. (25) thus takes the asymptotic form

$$
\begin{aligned}
\mu_{l}(n)=\tilde{g} n\left[\left(1+\kappa \frac{\tilde{b}}{\tilde{a}}+\frac{\tilde{a}}{\tilde{a}_{c r}}\right)\right. & +\frac{32}{3 \sqrt{\pi}} \frac{m^{*}}{m} \sqrt{n \tilde{a}^{3}} \\
& \left.+5 \tilde{R} \frac{m^{*}}{m} \sqrt{n \tilde{a}^{3}}\right] .
\end{aligned}
$$

In Eq. (31), the $(\kappa \tilde{b}) / \tilde{a}$ and $\tilde{a} / \tilde{a}_{c r}=-(0.24 \tilde{a} / d) \sqrt{m^{*} / m}$ [15] provide a further renormalization of the scattering length due to the random potential and optical lattice, i.e. $\tilde{g}_{\text {eff }}=\tilde{g}\left(1+\kappa \tilde{b} / \tilde{a}+\tilde{a} / \tilde{a}_{c r}\right)$; whereas, the remaining two terms proportional to the renormalized gas parameter generalize the LHY correction [14] to include effects of optical lattice and disorder. Eq. (31) therefore shows that the effects beyond mean field are much amplified via the introduction of periodic potential and external randomness, due to the increased inertia of mass $m^{*}$ along the direction of lattice and the interaction of bosons with impurities characterized by $\tilde{R}$. For vanishing disorder $\kappa=0$, Eq. (31) is reduced to the corresponding result in Ref. [15]; whereas for vanishing optical lattice $s=0$, our result recovers exactly the corresponding one in Ref. [17].

\section{Ground state density profile}

Using Eq. (25), one obtains by iteration the LDA ground state density profile $n(\mathbf{r})$ [5]

$$
n(\mathbf{r})=n_{T F}-n_{T F} k_{i n t}\left(n_{T F}\right)-n_{T F} k_{d i s}\left(n_{T F}\right),
$$

where

$$
n_{T F}(\mathbf{r})=n(0)-\frac{m}{2 \tilde{g}}\left(\omega_{x}^{2} x^{2}+\omega_{y}^{2} y^{2}+\omega_{z}^{2} z^{2}\right)
$$

is the mean-field value of density within the TF approximation. In Eq. (33), $n(0)$ is the lattice-renormalized TF density evaluated at the center of harmonic trap determined from

$$
n(0)=\frac{m}{2 \tilde{g}}\left(\omega_{x}^{2} X^{2}+\omega_{y}^{2} Y^{2}+\omega_{z}^{2} Z^{2}\right),
$$

where $X, Y, Z$ denote the size of the system. The second and third terms in Eq. (32) show that the quantum fluctuations due to interatomic interaction and disorder reduce the LDA total density, thereby fattening the cloud.

In the asymptotic 3D regime, $k_{\text {int }}$ in Eq. (26) and $k_{d i s}$ in Eq. (27) respectively approach

$$
k_{i n t}(\mathbf{r})=\frac{\tilde{a}}{\tilde{a}_{c r}}+\frac{32}{3 \sqrt{\pi}} \frac{m^{*}}{m}(n \tilde{a})^{\frac{3}{2}},
$$

and

$$
k_{d i s}(\mathbf{r})=\kappa \frac{\tilde{b}}{\tilde{a}}+5 \tilde{R} \frac{m^{*}}{m}(n \tilde{a})^{\frac{3}{2}} .
$$

It thus follows from Eq. (32) that the total density in the $3 \mathrm{D}$ limit takes the form

$n(\mathbf{r})=n_{T F}\left(1-\kappa \frac{\tilde{b}}{\tilde{a}}-\frac{\tilde{a}}{\tilde{a}_{c r}}\right)-\left(\frac{32}{3 \sqrt{\pi}}+5 \tilde{R}\right) \frac{m^{*}}{m}\left(\tilde{a} n_{T F}\right)^{\frac{3}{2}}$.

Eq. (37) shows that the effects beyond mean field due to both optical lattice and disorder have two consequences on the total density: first of all the TF density is further renormalized arising from the modified effective scattering length $n_{T F}\left(1-\kappa \tilde{b} / \tilde{a}-\tilde{a} / \tilde{a}_{c r}\right) \simeq \tilde{g} n_{T F} / \tilde{g}_{\text {eff }}=$ $n_{e f f}$; while the second point concerns the generalized LHY correction in the presence of disorder and optical lattice as exhibited by the last term in Eq. (37). For vanishing disorder and optical lattice, Eq. (37) is reduced to the corresponding one in Ref. [6].

It's important to mention here that the density $n(\mathbf{r})$ entering the hydrodynamic equations is the total density and shall not be confused with the condensate density $n_{c}(\mathbf{r})$ [6], particularly in cases where the effects beyond mean field are taken into account. In the asymptotic 3D regime, by extending the result of quantum depletion in [16] for an effectively uniform gas to that with harmonic trap using LDA, and together with Eq. (37) for the total density, one obtains the condensate density of the model system

$$
\begin{aligned}
n_{c}(\mathbf{r}) & =n_{T F}\left(1-\kappa \frac{\tilde{b}}{\tilde{a}}-\frac{\tilde{a}}{\tilde{a}_{c r}}\right) \\
& -\left[\frac{40}{3 \sqrt{\pi}}+\left(5+\frac{\sqrt{\pi}}{2}\right) \tilde{R}\right] \frac{m^{*}}{m}\left(\tilde{a} n_{T F}\right)^{\frac{3}{2}} .
\end{aligned}
$$

Comparison of Eqs. (37) and (38) shows that the effects beyond mean field reduce more condensate density than the total density. The $n(\mathbf{r})$ and $n_{c}(\mathbf{r})$ only equal in the limit where quantum depletions are negligible. In particular, Eq. (38) demonstrates that the disorder induced quantum depletion

$$
n_{R}(\mathbf{r})=\kappa \frac{\tilde{b}}{\tilde{a}} n_{T F}+\left(5+\frac{\sqrt{\pi}}{2}\right) \tilde{R} \frac{m^{*}}{m}\left(\tilde{a} n_{T F}\right)^{\frac{3}{2}}
$$

is considerably amplified at each point of the space, compared to the corresponding result in Ref. [11], when beyond-mean-field effects are included.

\section{Superfluid density profile}

The superfluid density is determined from $n_{s}(\mathbf{r})=$ $n(\mathbf{r})-n_{n}(\mathbf{r})$. Here, $n(\mathbf{r})$ is the total density determined from Eq. (32), while $n_{n}(\mathbf{r})$ is the normal fluid density. For a uniform gas in the absence of harmonic trap, the normal density $n_{n}$ can be determined from the longwavelength limit of the static transverse current-current response function $\chi_{T}(\mathbf{q})$ at $T=0$ [11, 27, 30, 33]

$$
n_{n}=\lim _{q \rightarrow 0} \chi_{T}(\mathbf{q}),
$$


where $\chi_{T}(\mathbf{q})$ can be derived within Bogliubov's theoretical framework [11, 12]. The result for $n_{n}$ of a BEC in the presence of weak disorder and a $2 \mathrm{D}$ optical lattice has been derived in our previous work [16] within the context of renormalized mass theory. Thus by extending the corresponding result in Ref. [16] to that with harmonic trap using LDA, we obtain

$$
n_{n}(\mathbf{r})=\frac{\tilde{R}}{4 \hbar d^{2}} \sqrt{2 m \tilde{g} n_{T F}} I\left(\frac{2 t}{\tilde{g} n_{T F}}\right) .
$$

Here, the $n_{T F}$ is the position-dependent TF density given by Eq. (33) and the function $I(x)$ with $x=2 t / \tilde{g} n_{T F}$ is defined as

$$
I(x)=\int_{-\pi}^{\pi} \frac{d^{2} \mathbf{k}}{(2 \pi)^{2}} \frac{1}{\sqrt{x \gamma(\mathbf{k})+2}[\sqrt{x}+\sqrt{x \gamma(\mathbf{k})+2}]^{2}}
$$

where $\gamma(\mathbf{k})=2-\cos \left(k_{x}\right)-\cos \left(k_{y}\right)$. Here, the parameter $x=2 t / \tilde{g} n_{T F}$ controls the dimensional crossover for nonuniform gases. In the limit of $3 \mathrm{D}$ regime corresponding to $x \gg 1, I(x)$ asymptotically approaches the function $\sqrt{2} /(6 \pi x)$. Accordingly, the LDA normal fluid density (41) takes the asymptotic form

$$
n_{n}(\mathbf{r})=\frac{2 \sqrt{\pi}}{3} \tilde{R} \frac{m^{*}}{m} n_{T F}\left(n_{T F} \tilde{a}^{3}\right)^{\frac{1}{2}} .
$$

However, with the disorder induced quantum depletion given by Eq. (39), the ratio $n_{n} / n_{R}$ at each point of the space departures from the factor $4 / 3$ pointed out in Ref. [1] , as a result of the effects beyond mean field.

\section{COLLECTIVE EXCITATIONS}

Having at hand all the quantities needed to solve Eq. (23), we can now proceed to investigate the low-energy collective excitations of the BEC's system under consideration. For this purpose, it's more advantageous to write $n_{s}=n-n_{n}$ and $\delta \mu=\left(\partial \delta \mu_{l} / \partial n_{T F}\right) \delta n$ in Eq. (23) with $\mu_{l}$ given by Eq. (25). After some elaborated algebra, we ultimately obtain

$$
\begin{aligned}
m \omega^{2} \delta n & +\tilde{\nabla} \cdot\left[n_{T F} \tilde{\nabla} \tilde{g} \delta n\right]=-\tilde{\nabla}^{2}\left(\tilde{g} n_{T F}^{2} \frac{\partial k_{i n t}}{\partial n_{T F}} \delta n\right) \\
& -\tilde{\nabla}^{2}\left(\tilde{g} n_{T F}^{2} \frac{\partial k_{d i s}}{\partial n_{T F}} \delta n\right)+\tilde{\nabla} \cdot\left[n_{n} \tilde{\nabla} \tilde{g} \delta n\right] \cdot
\end{aligned}
$$

Eq. (44) provides the appropriate generation of the zeroth order hydrodynamic equation in a $2 \mathrm{D}$ optical lattice [8]

$$
m \omega^{2} \delta n+\tilde{\nabla} \cdot\left[n_{T F} \tilde{\nabla} \tilde{g} \delta n\right]=0
$$

where the frequency $\omega$ can be solved for low-energy collective excitations within the mean-field approximation [5, 8]. After solutions to Eq. (45) are found, one can then solve Eq. (44) by treating all the terms on the right side as perturbation. Two types of such beyond-meanfield corrections can be identified: the first is associated with increased bulk compressibility $\chi^{-1}=\partial \mu / \partial n$ due to the enhanced quantum fluctuations caused by the combined effects of interatomic interaction, disorder and optical lattice [15]; the second type, by contrast, is unique of disorder which depletes superfluid fraction even at $T=0$.

Consequently, we follow Ref. [6] and obtain the analytical expression for the fractional shift in the frequency of low-lying collective modes

$$
\frac{\delta \omega}{\omega}=-\frac{\tilde{g}}{2 m \omega^{2}}\left\{\frac{\int d \mathbf{r} \tilde{\nabla}^{2} \delta n^{*}\left(n_{T F}^{2} \frac{\partial k_{i n t}}{\partial n_{T F}} \delta n\right)}{\int d \mathbf{r} \delta n^{*} \delta n}+\frac{\int d \mathbf{r}\left[\tilde{\nabla}^{2} \delta n^{*}\left(n_{T F}^{2} \frac{\partial k_{d i s}}{\partial n_{T F}} \delta n\right)+n_{n} \tilde{\nabla} \delta n^{*} \cdot \tilde{\nabla} \delta n\right]}{\int d \mathbf{r} \delta n^{*} \delta n}\right\}
$$

where $\omega$ is the frequency of collective modes obtained from Eq. (45) within the mean-field approximation [5].

Eq. (46) represents a major result of this paper. In what follows, we use this equation to derive some analytical results for phonon propagation and low energy collective modes.

\section{A. Phonon propagation}

An asymptotic analysis of Eq. (46) allows us to obtain the sound velocity of the model system in the $3 \mathrm{D}$ limit. Particularly, when the harmonic trap is absent, the model system is effectively uniform within the context of mass renormalization theory [8, 16]. As a result, the $n_{T F}, \partial k_{i n t} / \partial n_{T F}, \partial k_{d i s} / \partial n_{T F}$ and $n_{n}$ in Eq. (46) are position-independent and can be taken out of the integral. Together with Eqs. (35), (36) and (43), one obtains the asymptotic law for the frequency shift in Eq. (46) in 
the $3 \mathrm{D}$ regime

$$
\frac{\delta \omega}{\omega}=-\frac{\tilde{g}(\alpha+\beta+\gamma)}{4 m \omega^{2}} \frac{n_{T F}^{3 / 2} \int d \mathbf{r}\left(\tilde{\nabla}^{2} \delta n^{*}\right) \delta n}{\int d \mathbf{r} \delta n^{*} \delta n} .
$$

Here, $\alpha=\left(32 m^{*} / 3 \sqrt{\pi} m\right) \tilde{a}^{3 / 2}, \beta=\left(5 m^{*} / m\right) \tilde{R} \tilde{a}^{3 / 2}$ and $\gamma=-\left(4 \sqrt{\pi} m^{*} / 3 m\right) \tilde{R} \tilde{a}^{3 / 2}$. Meantime, solutions of Eq. (45) for this effectively uniform Bose gas have the form $\delta n \sim e^{i \mathbf{q} \cdot \mathbf{r}}$ with a phonon dispersion $\omega=c q$. Accordingly, we obtain the shift in the sound velocity

$$
\frac{\delta c}{c}=\frac{m^{*}}{m}\left[\frac{8}{\sqrt{\pi}}+\left(\frac{15}{4}-\sqrt{\pi}\right) \tilde{R}\right]\left(n_{T F} \tilde{a}^{3}\right)^{\frac{1}{2}}
$$

with respect to the lattice-modified Bogoliubov value $c=\sqrt{\tilde{g} n / m^{*}}$. The first term in Eq. (48) is a consequence of interatomic interaction, which generalizes the Beliaev result of $\delta c / c=8 \sqrt{n a^{3} / \pi}$ 34 for the sound velocity to include the effect of optical lattice. On the other hand, the remaining two terms $(15 / 4-\sqrt{\pi}) \tilde{R}\left(n_{T F} \tilde{a}^{3}\right)^{1 / 2}$ are proportional to $\tilde{R}$, representing the influence of weak disorder on phonon propagation. As is shown, disorder affects the sound velocity in two opposite ways: the first is related to quantum fluctuations that tend to increase sound speed, whereas the second is related to the depletion of superfluid fraction at $T=0$ which tends to suppress the phonon propagation. The former effect is also shared by interatomic interaction, while the latter, by contrast, is a unique consequence of disorder. In addition, all three terms in Eq. (48) demonstrate the same dependence on the gas parameter $n_{T F} \tilde{a}^{3}$ as the ground state chemical potential in Eq. (31). Eq. (48) therefore represents generalized LHY correction to the sound velocity in the presence of optical lattice and weak disorder.

It's worth mentioning here that one can also employ the compressibility-based definition for sound velocity $[20,21,26,35-37]$ to investigate the beyond-mean-fieldeffect induced shift. In our case, the normal component of fluid is pinned by the quenched disorder and doesn't directly participate in the propagation of the density disturbance. The corresponding definition for sound velocity typical of a superfluid in an optical lattice is therefore $m^{*} c^{2}=n_{s} \partial \mu / \partial n$ [26]. Here, the effect of disorder only enters indirectly through the compressibility $\chi^{-1}=\partial \mu / \partial n$ and the expression for $n_{s}$. To carry out the calculation beyond mean-field approximation, we consider the 3D asymptotic regime where the chemical potential approaches Eq. (31). The resulting sound velocity $c_{s}=c+\delta c$ is

$c_{s}=\sqrt{\frac{\tilde{g} n}{m^{*}}}\left\{1+\left[\frac{8}{\sqrt{\pi}}+\left(\frac{15}{4}-\frac{\sqrt{\pi}}{3}\right) \tilde{R}\right] \frac{m^{*}}{m}\left(n_{T F} \tilde{a}^{3}\right)^{\frac{1}{2}}\right\}$.

The corresponding fractional shift is $\delta c / c=[8 / \sqrt{\pi}+$ $(15 / 4-\sqrt{\pi} / 3) \tilde{R}]\left(m^{*} / m\right)\left(n_{T F} \tilde{a}^{3}\right)^{1 / 2}$. This result, however, is only consistent with Eq. (48) for varnishing disorder $(\tilde{R}=0)$. In this case, both results recover corresponding ones in Refs. [6] in free space and
[15] in the presence of optical lattice. Nonetheless, in the presence of disorder, the two differ by an amount $\left(2 \sqrt{\pi} m^{*} / 3 m\right) \tilde{R}\left(n_{T F} \tilde{a}^{3}\right)^{1 / 2}$. This inconsistency in the presence of disorder may be attributed to the pathology of perturbation theory and its failure (at finite order in an expansion in powers of $n_{T F} \tilde{a}^{3}$ ) to deal with the total density, condensate depletion and normal density in a completely consistent way.

\section{B. Collective modes}

Eq. (46) allows some direct analysis of respective roles played by interatomic interaction and disorder on the elementary excitations of a trapped BEC. The effects beyond mean field on low-lying collective modes have already been analyzed in free space [5], in the presence of optical lattice [8], and in the presence of disorder (but lattice free) [12], respectively. The following analysis presents a modest generalization of these previous results to the combined presence of optical lattice and weak disorder.

According to Eq. (46), interatomic interactions will not affect the frequencies of the so-called surface modes that satisfy $\widetilde{\nabla}^{2} \delta n^{*}=0$, despite the presence of optical lattice and disorder. In particular, the dipole mode corresponds to the center-of-mass motion of the superfluid and will not be influenced by the interaction, in accordance with the generalized Kohn's theorem [29]. Whereas, disorder will have an immediate effect on surface modes, shifting corresponding mean-field frequencies in Ref. [8] by an amount

$$
\frac{\delta \omega}{\omega}=-\frac{\tilde{g}}{2 m \omega^{2}} \frac{\int d \mathbf{r} n_{n} \tilde{\nabla} \delta n^{*} \cdot \tilde{\nabla} \delta n}{\int d \mathbf{r} \delta n^{*} \delta n} .
$$

In particular, the frequency shift in the dipole mode caused by disorder, as has been pointed out by Ref. [12], presents a departure from Kohn's theorem. Here, the result of Eq. (50) for dipole mode exhibits a magnified departure via the introduction of optical lattice. Such departure immediately distinguishes the beyond-meanfield effect due to disorder from that due to interatomic interaction. By contrast, the compressional modes will be affected by both disorder and interatomic interaction.

To explicitly observe the effects of disorder, interatomic interaction and optical lattice on low-lying collective modes, we consider a deformed harmonic trap in the form

$$
V_{h o}(\mathbf{r})=\frac{1}{2} m \omega_{\perp}^{2} r_{\perp}^{2}+\frac{1}{2} m \omega_{z}^{2} z^{2},
$$

where $r_{\perp}=\left(x^{2}+y^{2}\right)^{1 / 2}$ is the radial coordinate and $\omega_{\perp}$ is the radial trap frequency. The anisotropy is characterized by the parameter $\lambda=\omega_{z} / \omega_{\perp}$.

For such anisotropic trap, the zeroth order hydrodynamic Eq. (45) admits solutions $\delta n(\mathbf{r}) \sim \tilde{r}^{l} Y_{l m}(\theta, \phi)$ for 
$m= \pm l$ and $m= \pm(l-1)[5]$ that describes surface excitations, with $\tilde{r}=\sqrt{m^{*} / m}\left(x^{2}+y^{2}\right)^{1 / 2}$ being the latticerenormalized radial coordinate. The corresponding dispersion laws are given by $\omega(m= \pm l)=\sqrt{l m / m^{*}} \omega_{\perp}$ and $\omega(m= \pm(l-1))=\left[(l-1) m / m^{*} \omega_{\perp}^{2}+\omega_{z}^{2}\right]^{1 / 2}$. The periodic potential therefore effectively modifies the radial trap frequency $\omega_{\perp}$ by a factor $\sqrt{m / m^{*}}$, whereas leaving $\omega_{z}$ unaffected. The dipole modes and quadrupole modes are of particular experimental relevance. The lowest dipole mode $(l=1, m=0)$ describes the oscillation of the center of mass along the z-direction $\delta n(\mathbf{r}) \sim z$, with the zeroth order dispersion given by $\omega=\omega_{z}$ which is unaffected by the lattice. From Eq. (50), the result for the disorder induced frequency shift in the dipole mode is

$$
\frac{\delta \omega}{\omega}=-\frac{15}{16} \frac{\tilde{R}}{\hbar d^{2}} \sqrt{\frac{2 m \tilde{g}}{n(0)}} \int_{0}^{1} \rho^{2} \sqrt{1-\rho^{2}} I\left(\frac{y}{1-\rho^{2}}\right) d \rho,
$$

where the variable $y=2 t / \tilde{g} n(0)$ with $n(0)$ given by Eq. (34) and $I(x)$ is a function of variable $x=y /\left(1-\rho^{2}\right)$ given by Eq. (42). Eq. (52) shows that the frequency shift is directly proportional to the strength of disorder characterized by $\tilde{R}$. For varnishing disorder $(\tilde{R}=0)$, there is no frequency shift in the dipole modes, in conformity with Kohn's theorem [29]. For the quadrupole mode $\delta n(\widetilde{\mathbf{r}}) \sim \tilde{r}^{2} \sin ^{2} \theta e^{2 i \phi}$ with $l=2, m=2$, the meanfield dispersion is given by $\omega=\sqrt{2 m / m^{*}} \omega_{\perp}$. The corresponding frequency shift is found to be

$$
\frac{\delta \omega}{\omega}=-\frac{21}{8} \frac{\tilde{R}}{\hbar d^{2}} \sqrt{\frac{2 m \tilde{g}}{n(0)}} \int_{0}^{1} \rho^{4} \sqrt{1-\rho^{2}} I\left(\frac{y}{1-\rho^{2}}\right) d \rho,
$$

which also varnishes in the absence of disorder, consistent with our previous analysis.

Both Eqs. (52) and (53) exhibit a dependence on the parameter $y=2 t / \tilde{g} n(0)$ that characterizes the interplay between the kinetic energy in a lattice and the mean-field atom-atom interaction of a trapped gas. In the asymptotic $3 \mathrm{D}$ regime where $y \gg 1$, the function $I(x)$ with $x=y /\left(1-\rho^{2}\right)$ in Eq. (53) decays as $I(x) \simeq \sqrt{2} / 6 \pi x$. Accordingly, one finds the frequency shift in the dipole mode as $\delta \omega / \omega=$ $-\left(5 \pi^{3 / 2} m^{*} / 64 m\right) \tilde{R} \sqrt{\tilde{a}^{3} n(0)}$ and in the quadrupole mode as $\delta \omega / \omega=-\left(21 \pi^{3 / 2} m^{*} / 256 m\right) \tilde{R} \sqrt{\tilde{a}^{3} n(0)}$. Both results exhibit dependence on the gas parameter and disorder strength in a similar way as the normal density does in Eq. (43), which indicates that the peculiar role of disorder on the surface modes is closely related to the presence of normal component due to disorder.

To observe the effects beyond mean field on the compressional mode, we further assume an effective discshaped trap $\omega_{z} \gg \sqrt{m / m^{*}} \omega_{\perp}$ for simplicity. In this case, the zeroth order dispersion for the lowest compressional mode $(n=1, l=0)$ is given by $\omega=\sqrt{3} \omega_{z}$, corresponding to a density fluctuation $\delta n(\mathbf{r}) \sim z^{2}-2 \mu / 3 m \omega_{z}^{2}$. Note that this compressional mode is only determined by the trap frequency $\omega_{z}$, thereby unaffected by the lattice applied along the $x-y$ plane. It follows from Eq. (46) that the frequency shift in the mode $(n=1, l=0)$ is

$$
\frac{\delta \omega}{\omega}=\frac{21}{512} \frac{1}{\hbar d^{2}} \sqrt{\frac{2 m \tilde{g}}{n(0)}}[K(y)+\tilde{R}[(Z(y)-T(y)]] .
$$

Here, $y=2 t / \tilde{g} n(0)$, and $K(y)=(16 / \pi) y^{1 / 2} \int_{0}^{1} \rho^{2}\left(\rho^{2}-\right.$ 1) $D(x) d \rho$ with $x=y /\left(1-\rho^{2}\right)$ and $D(x)=x^{3 / 2} f^{\prime \prime}(x)-$ $3 f(x) / 4 \sqrt{x}$. Using the same definitions for variables $x$ and $y$, the functions $Z(y)$ and $T(y)$ are defined as $Z(y)=(8 \sqrt{2} / \pi) y^{1 / 2} \int_{0}^{1} \rho^{2}\left(\rho^{2}-1\right) G(x) d \rho$ where $G(x)=y^{3 / 2} Q^{\prime \prime}(x)-3 Q(x) / 4 \sqrt{x}$, and $T(y)=$ $32 \int_{0}^{1} \rho^{4} \sqrt{1-\rho^{2}} I(x) d \rho$ where $I(x)$ follows Eq. (42). Eq. (54) confirms our previous statement that the compressional mode is affected by both repulsive atom-atom interaction and disorder. Specifically, the first term in Eq. (54) represents the contribution from interatomic interaction, whereas the latter two are consequences of disorder. Moreover, Eq. (54) shows that disorder has two opposite effects on compressional excitations: the induced quantum fluctuation tends to increase excitation frequencies, whereas the depletion of superfluid fraction tends to decrease the excitation energy. This latter effect exhibited by the term proportional to $T(y)$, in particular, is unique of the effect of disorder on compressional modes. For vanishing disorder $\tilde{R}=0, \mathrm{Eq}$. (54) recovers the corresponding result in Ref. [15].

The parameter $y=2 t / \tilde{g} n(0)$ controls the dimensional crossover of the frequency shift in Eq. (54). In the asymptotic 3D regime corresponding to $y \gg 1$, functions $f(x), Q(x)$ and $I(x)$ with $x=y /\left(1-\rho^{2}\right)$ respectively decay as $f(x) \simeq 1.43 / \sqrt{x}-16 \sqrt{2} / 15 \pi x$, $Q(x) \simeq-1 / \sqrt{\pi} x$ and $I(x)=\sqrt{2} / 6 \pi x$. Hence Eq. (54) asymptotically approaches $\delta \omega / \omega=$ $35 \sqrt{\pi} m^{*} / 128 m[1-3 / 5 \tilde{R}(25 \sqrt{\pi} / 64-\pi / 8)] \sqrt{\tilde{a}^{3} n(0)}$. This result provides a further generalization by disorder of the frequency shift caused by the lattice-generalized LHY correction in Ref. [15]. Moreover, this result shows that the disorder-induced frequency shift is of the same order of magnitude, but with an opposite sign, compared to that due to atom-atom interaction.

\section{POSSIBLE EXPERIMENTAL SCENARIOS AND CONCLUSION}

Central to test the validity of the hydrodynamic picture proposed in this paper are the experimental abilities to measure the superfluid density and condensate density respectively. For a gaseous BEC, the condensate fraction is readily measured through mapping of occupation numbers in the momentum space to real space by expansion imaging [1]. Whereas, to determine the superfluid fraction, one can use a method proposed in Ref. [38]. There, a BEC is beard to an optically-induced vector potential, 
which simulates a uniform rotation of BEC. As a result, the normal fluid picks up non-zero angular momentum, whereas the superfluid acquires no angular momentum. Thus the spectroscopy can be used to measure the net change in the angular momentum of the fluid, and therefore the superfluid fraction.

Another difficulty that may arise is how to experimentally measure the beyond-mean-field correction to the equation of state due to the interatomic interaction and disorder. The concern is as follows [39]: for a harmonic trap, the quantum depletion cannot be observed during the ballistic expansion in the typical Thomas-regime. Because the mean-field energy is much larger than the trap frequency, the cloud remains locally adiabatic during the expansion. The condensate at high density transforms adiabatically into a condensate at low density with diminishing quantum depletion. The above concern is true for a harmonically trapped BEC. However, it can be ruled out by introducing an optical lattice. In general, the confinement frequency at each lattice site far exceeds the interaction energy, and the time-of-flight images are essentially a snapshot of the momentum distribution at the time of the lattice switch-off, thus allowing for a direct observation of the quantum depletion.

Upon overcoming the above two difficulties, the experimental realization of our scenario amounts to controlling three parameters whose interplay underlies the physics of this work: the strength of an optical lattice $s$, the mean-field interaction between bosonic atoms $\tilde{g} n_{T F}$, and the strength of disorder $\tilde{R}$. All these quantities are experimentally controllable using state-of-the-art technologies. The interatomic interaction can be controlled in a very versatile manner via the technology of Feshbach resonances [40]. In the typical experiments to date, the values of ratio $\tilde{g} n_{T F} / E_{R}$ range from 0.02 to 1 [2, 3]. The depth of an optical lattice $s$ can be changed from $0 E_{R}$ to $32 E_{R}$ almost at will [41]. The above scenario for disorder can be realized in cold atomic systems using several methods in a controlled way. They include applying optical potentials created by laser speckles or multi-chromatic lattices [42 45], introducing impurity atoms in the sample [46] and manipulating the collision between atoms [47]. Therefore, the phenomena discussed in this paper should be observable within the current experimental capability. We emphasize here that the work presented in this paper is restricted to weak disorder and weak interatomic interaction. For further investigations in the presence of stronger inter-atomic interaction or disorder, the path-integral Monte Carlo simulation is a reliable method [48].

In summary, we explore the combined effects of weak disorder and a 2D optical lattice on the collective excitations of a harmonically trapped BEC. Accordingly, we extend the hydrodynamic equations for superfluid [8] for a 2D optical lattice to include the effects of weak disorder. For small amplitude excitations, the corresponding equations are characterized by the disorder-depleted superfluid fluid density, a renormalized interatomic interaction and an effective mass capturing the effect of periodic potential. Using these hydrodynamic equations, we calculate the collective frequencies beyond the mean-field approximation. Our results reveal peculiar role of disorder, interplaying with optical lattice and atom-atom interactions, on the elementary excitations of a trapped BEC. In particular, we present detailed analysis for consequences of disorder on phonon propagation and lattice-modified surface modes. Conditions for possible experimental realization of our scenario are also proposed.

Acknowledgements We thank Biao Wu for helpful discussions. $\mathrm{YH}$ and $\mathrm{BBH}$ are supported by the Hongkong Research Council (RGC) and the Hong Kong Baptist University Faculty Research Grant (FRG). ZXL is supported by the IMR SYNL-TS Ke Research Grant.
[1] F. Dalfovo, S. Giorgini, L. P. Pitaevskii, and S. Stringari, Rev. Mod. Phys. 71, 463 (1999).

[2] O. Morsch and M. Oberthaler, Rev. Mod. Phys. 78, 179 (2006).

[3] I. Bloch, J. Dalibard and W. Zwerger,Rev. Mod. Phys. 80, 885 (2008).

[4] V. I. Yukalov, Laser Phys. 19, 1 (2009).

[5] S. Stringari, Phys. Rev. Lett. 77, 2360 (1996).

[6] L. P. Pitaevskii and S. Stringari, Phys. Rev. lett. 81, 4541 (1998).

[7] E. Braaten and J. Pearson, Phys. Rev. Lett. 82, 255 (1999).

[8] M. Krämer, L. P. Pitaevskii, and S. Stringari, Phys. Rev. Lett. 88, 180404 (2002).

[9] R. C. Kuhn, C. Miniatura, D. Delande, O. Sigwarth, and C. A. Müller, Phys. Rev. Lett. 95, 250403 (2005); H. Gimperlein, S. Wessel, J. Schmiedmayer, and L. Santos, Phys. Rev. Lett. 95, 170401 (2005); L. Sanchez-Palencia,
D. Clément, P. Lugan, P. Bouyer, G.V. Shlyapnikov, and A. Aspect, Phys. Rev. Lett. 98, 210401 (2007); D. Delande and J. Zakrzewski, Phys. Rev. Lett. 102, 085301 (2009); S. Pilati, S. Giorgini, and N. Prokof'ev, Phys. Rev. Lett. 102, 150402 (2009); L. Fontanesi, M. Wouters, and V. Savona, Phys. Rev. Lett. 103, 030403 (2009); L. Dang, M. Boninsegni, and L. Pollet, Phys. Rev. B 79, 214529 (2009); G. M. Falco, T. Nattermann, and V. L. Pokrovsky, Europhys. Lett. 85, 30002 (2009).

[10] D. W. Wang, M. D. Lukin, and E. Demler, Phys. Rev. Lett. 92, 076802 (2004).

[11] Kerson Huang and Hsin-Fei Meng, Phys. Rev. Lett. 69, 644 (1992); A. V. Lopatin and V. M. Vinokur, Phys. Rev. Lett. 88, 235503 (2002).

[12] G. M. Falco, A. Pelster, and R. Graham, Phys. Rev. A 76, 013624 (2007).

[13] L. Sanchez-Palencia and M. Lewenstein, Nat. Phys. 6, 87 (2010). 
[14] T. D. Lee and C. N. Yang, Phys. Rev. 105, 1119 (1957); T. D. Lee, K. W. Huang, and C. N. Yang, Phys. Rev. 106, 1135 (1957).

[15] G. Orso, C. Menotti, and S. Stringari, Phys. Rev. lett. 97, 190408 (2006).

[16] Ying Hu, Z. X. Liang, and B. B. Hu, Phys. Rev. A 80, 043629 (2009).

[17] G. E. Astrakharchik, J. Boronat, J. Casulleras, and S. Giorgini, Phys. Rev. A 66, 023603 (2002).

[18] M. Wouters and G. Orso, Phys. Rev. A 73, 012707 (2006).

[19] P. Pedri and L. Santos, Phys. Rev. Lett. 91, 110401 (2003).

[20] M. Krämer, C. Menotti, L. P. Pitaevskii, and S. Stringari, Eur. Phys. J. D 27, 247 (2003)

[21] Z. X. Liang, X. Dong, Z. D. Zhang, and Biao Wu, Phys. Rew. A 78, 023622 (2008).

[22] C. Fort, F. S. Cataliotti, L. Fallani, F. Ferlaino, P. Maddaloni, and M. Inguscio, Phys. Rev. Lett. 90, 140405 (2003).

[23] H. Moritz, T. Stöferle, M. Köhl, and T. Esslinger, Phys. Rev. Lett. 91, 250402 (2003).

[24] L. D. Landau, J. Phys. (USSR) 5, 71 (1941).

[25] I. M. Khalatnikov, An Introduction to the Theory of Superfluidity (Benjamin, New York, 1965).

[26] S. Giorgini, L. P. Pitaevskii, and S. Stringari, Phys. Rev. B 49, 12938 (1994).

[27] D. Forster, Hydrodynamic Fluctuations, Broken Symmetry, and Correlations Functions (Benjamin, Reading, MA, 1975).

[28] V. I. Yukalov and E. P. Yukalova, Phys. Rev. A 74, 063623 (2006); V. I. Yukalov, E. P. Yukalova, K. V. Krutitsky, and R. Graham, ibid. 76, 053623 (2007).

[29] L. P. Pitaevskii and S. Stringari, Bose-Einstein Condensation (Clarendon Press, Oxford, 2003).

[30] D. Pines and P. Noziéres, The Theory of Quantum Liquids, (Benjamin, New York, 1966), Vol. I; P. Noziéres and D. Pines, The Theory of Quatum Liquids, (AddisonWesley, Reading, MA, 1990), Vol. II.
[31] D. Zwillinger, Standard Mathematical Tables and Formulae, (CRC Press, Boston, 1996).

[32] E. H. Lieb and W. Liniger, Phys. Rev. 130, 1605 (1963); E. H. Lieb, ibid. 130, 1616 (1963).

[33] G. Baym, Mathematical Methods in Solid State and Superfluid Theory, edited by R. C. Clark and G. H. Derrick (Oliver and Boyd, Edinburgh, 1969). p. 151.

[34] S. T. Beliaev, Sov. Phys. JETP 34, 299 (1958).

[35] E. Taylor and E. Zaremba, Phys. Rev. A 68, 053611 (2003).

[36] C. Menotti, M. Krämer, A. Smerzi, L. Pitaevskii and S. Stringari, Phys. Rev. A 70, 023609 (2004).

[37] J. P. Martikainen and H. T. C. Stoof, Phys. Rev. A 69, 023608 (2004).

[38] N. R. Cooper and Z. Hadzibabic, Phys. Rev. Lett. 104, 030401 (2010); I. Carusotto, Physics 3, 5 (2010).

[39] K. Xu, Y. Liu, D. E. Miller, J.K. Chin, W. Setiawan, and W. Ketterle, Phys. Rev. Lett. 96, 180405 (2006).

[40] E. Tiesinga, B.J. Verhaar, H.T.C. Stoof, Phys. Rev. A. 47, 4114 (1993); S. Inouye et al., Nature (London) 392, 151 (1998).

[41] M. Greiner, O. Mandel, T. Esslinger, T. W. Hansch, and J. Bloch, Nature (London) 415, 39 (2002).

[42] B. Damski, J. Zakrzewski, L. Santos, P. Zoller, and M. Lewenstein, Phys. Rev. Lett. 91, 080403 (2003).

[43] R. Roth and K. Burnett, Phys. Rev. A. 68, 023604 (2003).

[44] J. E. Lye, L. Fallani, M. Modugno, D. S. Wiersma, C. Fort, and M. Inguscio, Phys. Rev. Lett. 95, 070401 (2005).

[45] M. White, M. Pasienski, D. McKay, S. Q. Zhou, D. Ceperley, and B. DeMarco, Phys. Rev. Lett. 102, 055301 (2009).

[46] S. Ospelkaus, C. Ospelkaus, O. Wille, M. Succo, P. Ernst, K. Sengstock, and K. Bongs, Phys. Rev. Lett. 96, 180403 (2006).

[47] U. Gavish and Y. Castin, Phys. Rev. Lett. 95, 020401 (2005).

[48] S. Pilati, S. Giorgini, M. Modugno, and N. Prokof'ev, e-print arXiv:1002.3704. 